\title{
ACRL candidates, 1991 elections
}

\section{Who's who on the Spring ballot.}

$\mathbf{T}$ he listing for each of the following candidates includes their title, institution, and institutional address.

\section{Vice-President/President-Elect}

Jacquelyn M. Morris, College Librarian, Occidental College Library, 1600 Campus Road, Los Angeles, CA 90041; John C. Tyson, State Librarian, Virginia State Library, 11th Street at Capitol Square, Richmond, VA 23219.

\section{Board of Directors}

Director-at-Large: Ray E. Metz, Assistant Director for Systems, Case Western Reserve University, Freiberger Library, 11161 East Boulevard, Cleveland, OH 44106; Victoria A. Montavon, Library Director, Saint Joseph's University, 5600 City Ave., Philadelphia, PA 19131-1395.

Director-at-Large: David L. Easterbrook, Principal Bibliographer, University of Illinois at Chicago, University Library, 801 South Morgan Street, P.O. Box 8198/MC 234, Chicago, IL 60680; Linda L. Phillips, Head, Cooperative Information Services, University of Tennessee, John C. Hodges Library, 1015 Volunteer Boulevard, Knoxville, TN 37996-1000.

\section{Afro-American Studies Librarian Section (AFAS)}

Vice-Chair/Chair-Elect: Doris H. Clack, Professor, Florida State University, School of Library and Information Studies, Tallahassee, FL 32306; Alice Reviere Smith, Black Studies/Women Studies Librarian, Cleveland State University Library, 1860 E. 22nd Street, Cleveland, OH 44115.

Secretary: Dorice L. Horne, Computer/Media Librarian, New York University, Medical Library, 550 First Avenue, New York, NY 10016; Joyce E.
Jelks, Assistant Manager/Assistant Curator, Atlanta-Fulton Public Library, 1 Margaret Mitchell Square NW, Atlanta, GA 30303-1089.

Member-at-Large: Luella B. Davis, Bibliographic Instruction Coordinator, Emory University, Robert W. Woodruff Library, Reference Department, Atlanta, GA 30322; Marcelle E. Hughes, Associate Librarian, University of Cincinnati, Langsam Library, Cincinnati, OH 452210033.

\section{Anthropology and Sociology Section (ANSS)}

Vice-Chair/Chair-Elect: Maija Lutz, Head of Technical Services/Collection Development Librarian, Harvard University, Tozzer Library, 21 Divinity Avenue, Cambridge, MA 02138; James Williams, Assistant Education and Social Science Librarian, University of Illinois Library, $1408 \mathrm{~W}$. Gregory Drive, Urbana, IL 61801.

Secretary: Joyce Ogburn, Head, Ordering Section, The Pennsylvania State University, E506 Pattee Library, University Park, PA 16802.

Member-at-Large: Pauline D. Manaka, Social Science Librarian, University of California-Irvine, General Library, Irvine, CA 92713; Nancy Skipper, Reference Librarian/Anthropology Bibliographer, Cornell University, 201 Olin Library, Ithaca, NY 14853-5301.

\section{Arts Section (ARTS)}

Vice-Chair/Chair-Elect:William F. Coscarelli, Department Head Fine Arts/Media, University of Georgia Libraries, Athens, GA 30602; Micheline C. Nilsen, Slide Librarian, University of Pennsylvania, Philadelphia, PA 19104-6311.

Secretary: Joan F. Cheverie, Head, Government Documents, Georgetown University, Joseph Mark Lauinger Library, P.O. Box 37445, Washing- 
ton, DC 20013-7445; Carolyn A. Sheehy, Director, North Central College Library, Oesterie Library, 320 East School Street, Naperville, IL 60540.

\section{Asian and African Section (AAS)}

Vice-Chair/Chair-Elect: Ray P. Boylan, Director, Collection Resources, Center for Research Libraries, 6050 S. Kenwood Avenue, Chicago, IL 60637; David G. Hirsch, Jewish Studies Bibliographer, University Research Library, University of California, Los Angeles, CA 90024-1575.

Secretary: Elise L. Chin, Acting Head, East Asia Library, University of Washington, Seattle, WA 98195; Lee S. Dutton, Librarian, D. Hart Southeast Asia Collection, University Libraries, Northern Illinois University, DeKalb, IL 601152868.

Member-at-Large: Gregory A. Finnegan, Reference Librarian, Dartmouth College Library, Hanover, NH 03755; Christine H. Guyonneau, Reference Librarian, University of Indianapolis, Krannert Memorial Library, 1400 East Hanna Avenue, Indianapolis, IN 46227; Karl K. Kahler, East Asian Bibliographer, University of Pennsylvania, Van Pelt-Dietrich Library, 3420 Walnut Street, Philadelphia, PA 19104-6206; Joseph J. Lauer, Africana Bibliographer, Michigan State University, East Lansing, MI 48824-1034.

\section{Bibliographic Instruction Section (BIS)}

Vice Chair/Chair Elect: Ellen Broidy, Coordinator of Library Education Services, University of California-Irvine Library, P.O. Box 19557, Irvine, CA 92713; Sharon B. Mader, Associate Director for Information and Research, DePaul University Libraries, 2323 North Seminary Avenue, Chicago, IL 60614 .

Secretary: Sara Penhale, Science Librarian, Earlham College, Wildman Science Library, Richmond, IN 47374; Robert F. Rose, Assistant Director for Informational and Instructional Services, University of Northern Iowa, Donald O. Rod Library, Cedar Falls, IA 50613-3675.

Member-at-Large: Linda S. Muroi, Reference Librarian, San Diego State University Library, 5300 Campanile Drive, San Diego, CA 921820511; Loanne L. Snavely, Reference Librarian/ Subject Specialist, Bloomsburg University, Harvey A. Andruss Library, Bloomsburg, PA 17815.

\section{College Libraries Section (CLS)}

Vice Chair/Chair-Elect: Mignon S. Adams, Director of Library Services, Philadelphia College of Pharmacy and Science, Joseph W. England Library, 42nd Street and Woodland Avenue, Philadelphia, PA 19104-4491; Judith Gibson Green,
University Librarian, Colgate University, Everett Needham Case Library, Hamilton, NY 13346.

Secretary: Richard J. Kuhta, University Librarian, Saint Lawrence University, Owen D. Young Library, Park Street, Canton, NY 13617; Larry R. Oberg, Director of Libraries, Albion College, Stockwell-Mudd Libraries, 602 E. Cass Street, Albion, MI 49224.

Member-at-Large: Stephanie R. Bangert, Library Director, St. Mary's College of California, P.O. Box 5150, Moraga, CA 94575; Mary K. Sellen, Library Director, Chapman College, Clarke Memorial Library, 333 N. Glassell Street, Orange, CA 92666.

\section{Community and Junior College Libraries Section (CJCLS)}

Vice-Chair/Chair-Elect: Susan M. Maltese, Cataloging Librarian, Oakton Community College Library, 1600 E. Golf Road, Des Plaines, IL 60016; Derrie B. Roark, District Dean of Learning Resources, Hillsborough Community College, Ybor City Campus, Tampa, FL 33675-5096.

Secretary: Lenora C. Lockett, Library Director, Delgado Community College, Moss Memorial Library, 615 City Park Avenue, New Orleans, LA 70119; V. Sue Hatfield, Assistant Director of Library Services, DeKalb College, Central Campus Library, 55 N. Indian Creek, Clarkston, GA 30021.

\section{Education and Behavioral Sciences Section (EBSS)}

Vice-Chair/Chair-Elect: Jo Ann Carr, Director, IMC, University of Wisconsin, 225 N. Mills, Madison, WI 53706; Sally Willson Weimer, Reference Librarian, University of California, UCSB Library, Santa Barbara, CA 93106.

Secretary: Pam M. Baxter, Psychological Sciences Librarian, Purdue University Libraries, West Lafayette, IN 47907; Kathleen M. McGowan, Coordinator of Reference Services and Collections/Education Bibliographer, University of Rochester, Rush Rhees Library, Rochester, NY 14627.

\section{Law and Political Science Section (LPSS)}

Vice-Chair/Chair-Elect: Charles D. Spornick, Collection Management Coordinator, Emory University, General Libraries, Atlanta, GA 30322; Caroline J. Tibbetts, Associate Librarian, University of Delaware, Morris Library, Newark, DE 19717-5267.

Secretary: James W. Hart, Head of Public Services, University of Cincinnati, Cincinnati, $\mathrm{OH}$ 45221-0033; Graham R. Walden, Assistant Profes- 
sor/Reference Librarian, Ohio State University Libraries, 1858 Neil Avenue Mall, Columbus, $\mathrm{OH}$ 43210-1286.

Member-at-Large: Elizabeth A. Sibley, Librarian, University of California-Berkeley, Moffitt Undergraduate Library, Berkeley, CA 94720; Phillip W. Wilkin, Social Science Bibliographer, University of Pittsburgh Libraries, Hillman Library, Pittsburgh, PA 15260.

\section{Rare Books and Manuscripts Section} (RBMS)

Vice Chair/Chair-Elect: Gary L. Menges, Head, Special Collections and Preservation Division, University of Washington, Suzzallo Library, FM25, Seattle, WA 98195; Michael T. Ryan, Director of Library Collections/Curator of Special Collections, Stanford University, Stanford, CA 943056004.

Secretary: Elizabeth L. Johnson, Head of Technical Services, Indiana University, Lilly Library, Bloomington, IN 47405; Janice E. Matthiesen, Special Collections Cataloger, University of California-Los Angeles, University Research Library, 405 Hilgard Avenue, Los Angeles, CA 90024.

Member-at-Large: Nancy H. Burkett, Associate Librarian, American Antiquarian Society, 185 Salisbury Street, Worcester, MA 01609-1634; Kathryn N. Morgan, Curator of Rare Books, University of Virginia, Alderman Library, Charlottesville, VA 22903.

\section{Science and Technology Section (STS)}

Vice Chair/Chair-Elect: Karen E. Feeney, Head, Science and Engineering Library, University of California-San Diego, Science and Engineering Library, La Jolla, CA 92093; Susanne J. Redalje, Head Chemistry Library, University of Washington, Chemistry Library, BG-10, Seattle, WA 98195.

Secretary: Helen E. Gbala, Head, Monographs Department, Illinois Institute of Technology, Paul V. Galvin Library, 35 West 33rd Street, Chicago, IL 60616; Lois M. Pausch, Acting Geology Librarian/Assistant Mathematics Librarian, University of Illinois at Urbana Champaign, $1301 \mathrm{~W}$. Gregory Drive, Urbana, IL 61801.

\section{Slavic and East European Section (SEES)}

Vice Chair/Chair-Elect: Molly F. Molloy, Slavic Reference Librarian, Hoover Institution on War, Revolution and Peace Library, Stanford University, Stanford, CA 94305-6010.

Member-at-Large: Susan K. Burke, Assistant
Slavic Librarian, University of Washington, Suzzallo Library, FM-25, Seattle, WA 98195; Sandra L. Levy, Assistant Slavic Librarian, University of Chicago Library, Joseph Regenstein Library, 1100 E. 57th Street, Chicago, IL 60637-1502.

\section{University Libraries Section (ULS)}

Vice Chair/Chair-Elect: James R. Martin, University Librarian, University of Southern Mississippi, Southern Station, Hattiesburg, MS 39406; Carolyn L. Robison, Associate University Librarian/Professor, Georgia State University, Pullen Library, 100 Decatur Street SE, Atlanta, GA 30303-3081.

Secretary: Lori A. Goetsch, Head, Information/Reference, Michigan State University Libraries, East Lansing, MI 48824-1034; Joan M. Repp, Director of Access Service, Bowling Green State University, Jerome Library, Bowling Green, $\mathrm{OH}$ 43403.

Member-at-Large (3-year term): William J. Crowe, Dean of Libraries, University of Kansas, Lawrence, KS 66045-2800; Paul M. Gherman, Director of Libraries, Virginia Polytechnic Institute and State University, University Libraries, Blacksburg, VA 24061-0434; George R. Jaramillo, Director of Public Services and Personnel, University of Northern Colorado, James A. Michener Library, Greeley, CO 80639; David W. Lewis, Head, Research and Information Services Department, University of Connecticut, Homer Bagbbidge Library, Box U-5R, Storrs, CT 06269.

Member-at-Large (2-year term): Martin $P$. Courtois, Database Coordinator/Reference Librarian, Michigan State University Libraries, East Lansing, MI 48824-1034; James A. Damico, Director of University Libraries, University of South Alabama Library, 307 University Boulevard, Mobile, AL 36688; Jill B. Fatzer, Dean of Library Services, University of New Orleans, Earl K. Long Library, Lake Front, New Orleans, LA 70148; Judy A. Sackett, Head, Preservation Department, University of Kentucky Libraries, Lexington, KY 40506-0039.

Member-at-Large (1-year term): Stella Bentley, Assistant University Librarian/Collection Development, University of California, UCSB Library 3589, Santa Barbara, CA 93106; Doris Anne Bradley, Serials Catalog Librarian, University of North Carolina at Charolotte, J. Murrey Atkins Library, Charlotte, NC 28223; Patricia L. Bril, Acting Associate University Librarian, California State University, P.O. Box 4150, Fullerton, CA 92634; Doug Phelps, Director of General Technical Services, Vanderbilt University Library, Jean and Alexander Heard Library, 419 21st Avenue South, Nashville, TN 37240-0007. 


\section{Western European Specialists Section (WESS)}

Vice Chair/Chair-Elect: Ceres B. Birkhead, Coordinator Library Instruction/Coordinator Humanities Selectors, University of Utah, Marriott Library, Salt Lake City, UT 84112-1179; Danielle Mihram, Head, Reference, University of Southern California, Doheny Memorial Library, University Park, Los Angeles, CA 90089-0182.

Secretary: James W. Romer, Head, Acquisitions Librarian, University of North Carolina, Walter Clinton Jackson Library, 1000 Spring Garden Street, Greensboro, NC 27412-5201; Rebecca A. Stuhr-Rommereim, Deputy Reader Services/ Librarian, Humanities Bibliographer, Grinnell College, Burling Library, P.O. Box 805, Grinnell, IA 50112-0811.

Member-at-Large: Carol D. Cooper, Vice President, International Publishing Operations, K.G. Saur, 245 W. 17th Street, New York, NY 10011; Thomas D. Kilton, Assistant Modern Languages and Linguistics Librarian/Associate Professor of Library Administration, University of Illinois
Library, 1408 W. Gregory Drive, Urbana, IL 61801.

\section{Women's Studies Section (WSS)}

Vice Chair/Chair-Elect: Virginia L. Daley, Women's Studies Archivist, Duke University, William R. Perkins Library, Durham, NC 27706; Betty J. Glass, Instructional Services Librarian, University of Nevada-Reno, Noble H. Getchell Library, Reno, NV 89557.

Secretary: Cynthia S. Faries, Senior Assistant Reference Librarian, Pennsylvania State University, Pattee Library, University Park, PA 16802; Bernice K. Lacks, Head of Reference, California State University-Fresno, Henry Madden Library, Fresno, CA 93740-0034.

Member-at-Large: Argent Sue Gibson, Division Head of Collection Management and Development, Atlanta University Center, Robert W. Woodruff Library, 111 James P. Brawley Drive SW, Atlanta, GA 30314; Nancy B. Luikart, Information Access Librarian for Reference Services, Texas Tech University Libraries, Lubbock, TX 79409-0002.

\section{The future of reference III:}

\section{An introduction}

\section{A panel discussion held at the University of Texas at Austin, Spring 1990.}

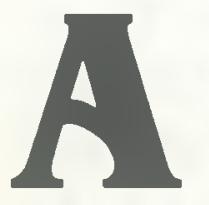

third program on the future of reference, "Paradigm Shift for Information Service; Business-But Not as Usual," was held at The University of Texas at Austin General Libraries during the Spring of 1990. Where the previous program (see C\&RL News, October 1989, pp. 780-799) examined an expansion of reference services to include an entirely new paradigm, or model of service, the third program considered the structure of services and the changes in organizational experiences in the electronic environment.

The program was sponsored by the General Libraries' Reference and Information Services Committee. The attendees included academic librarians and administrators from the General Libraries and the Tarlton Law Library, librarians from the Central Texas area, faculty and students of the Graduate School of Library and Information 